\title{
Estrategias metacognitivas, motivación académica y rendimiento académico en alumnos ingresantes a una universidad de Lima metropolitana
}

\author{
Gaby Thornberry Noriega
}

Se estudió la relación entre el uso de estrategias metacognitivas, la motivación académica y el rendimiento académico en estudiantes universitarios. La muestra estuvo compuesta por 116 alumnos ingresantes al primer ciclo de estudios de una universidad privada en Lima metropolitana. Se elaboró la Escala de Motivación Académica, instrumento de tipo cuestionario en el que se evalúan las dimensiones motivacionales de autoeficacia académica, atribuciones causales y motivación de logro. Los resultados revelaron que existen correlaciones positivas entre el rendimiento académico y las estrategias metacognitivas, pero esta relación no logra ser predictiva. En cambio, la motivación académica logra predecir el rendimiento en un $12,6 \%$.

estrategias metacognitivas / motivación académica / autoeficacia / atribuciones causales

Metacognitive strategies, academic motivation and academic performance in entering health science students in a university in Lima.

The present research studied the relationship between the use of metacognitive strategies, academic motivation and academic achievement in university students. The sample was composed by 116 first semester students, of a private university in Lima Metropolitana. The Scale for Academic Motivation was created to asses the motivational dimensions of: Academic Self-efficacy, Causal Attributions and Achievement Oriented Actions. Positive correlations between metacognitive strategies and academic achievement were found, although results were not predictive. Instead, academic motivation was found to predict achievement in 12,6\%.

metacognitive strategies / academic motivation / self-efficacy / causal attribution 


\section{INTRODUCCIÓN}

Desde hace mucho tiempo, psicólogos y educadores en el mundo entero han llevado a cabo investigaciones para intentar identificar aquello que diferencia a los estudiantes con un buen rendimiento académico de aquellos que presentan dificultades. Estos esfuerzos de investigación han encontrado una serie de variables que influyen en el logro del aprendizaje, desde variables internas al individuo como su inteligencia, aptitudes, motivación, hasta variables externas como la estimulación temprana los métodos educativos, los materiales.

Hoy en día encontramos que psicólogos y educadores tienen la mira en los aportes de la metacognición en el éxito académico de los estudiantes (Chrobak, 2004; Muria, 2004; García, 2001; Flores, 2000; Romanville, 1994), pero debido a las escasas investigaciones respecto al tema, poco se conoce a ciencia cierta en nuestro medio. Investigaciones extranjeras muestran la importancia de la motivación como factor mediador de la puesta en práctica de las estrategias metacognitivas. Es a partir de este vacío en la información que se propone la presente investigación, en la que se buscó evidenciar la relación entre el uso de estrategias metacognitivas, la motivación académica y el rendimiento académico en estudiantes ingresantes a una universidad de Lima metropolitana. Se optó por trabajar con estudiantes ingresantes a la universidad con el fin de observar el nivel tanto metacognitivo como motivacional con que llega esta población a iniciar sus estudios profesionales.

En el ámbito global, la presente investigación intenta contribuir a la comprensión de un problema socioeducativo cada vez más evidente y generalizado a escala mundial: la insuficiente capacidad de autorregulación y motivación de muchos aprendices y, en consecuencia, su insatisfactorio rendimiento académico universitario. A escala regional, y debido a que se trata de uno de los primeros estudios llevados a cabo en el país sobre este tema, la presente investigación busca dar luces sobre la manera en que se presentan las estrategias metacognitivas y motivacionales en su relación con el rendimiento académico, en los alumnos de primeros ciclos universitarios. La información desprendida de esta búsqueda es de gran utilidad para comprender el estado actual de nuestros estudiantes universitarios y sus necesidades de apoyo frente a las situaciones de aprendizaje.

Hace un par de décadas, un grupo de investigadores empezó a observar un fenómeno difícil de explicar: encontraron que algunos alumnos de inteligencia normal, que contaban con los estímulos necesarios y conocían estrategias cognitivas para aplicar en su estudio, no lograban un rendimiento académico exitoso (Sandia, 2004; Kuhl, 2001; 
Meltzer, 1993; Wong, 1992). Empezaron a notar que las características más saltantes entre los alumnos con dificultades en el aprendizaje era su desorganización, falta de atención a la tarea y su pasividad. Encontraron que su dificultad nacía de una inadecuada dirección y manejo de sus estrategias cognitivas, para orientarlas, de manera efectiva y flexible, hacia las metas. A esta destreza para pensar con respecto al propio pensamiento la llamaron metacognición.

Hoy en día los autores coinciden en conceptualizar la metacognición como la toma de conciencia del propio pensamiento y el control sobre este (Mayer, 2003). Dicho constructo incluye las estrategias de planificar antes de ejecutar una tarea, monitorear su comprensión y su ejecución y chequear el resultado luego de haber terminado. Así, mientras las estrategias cognitivas manejan el aprendizaje, las estrategias metacognitivas controlan, organizan y reflexionan sobre dicho proceso de aprendizaje (Mayer, 2003; Covington, 2000).

Se han desarrollado muchas investigaciones con respecto a la relación entre las estrategias metacognitivas y el rendimiento académico (Winne, 2005; Torgesen, 1994; Meltzer, 1993). Los resultados de estos estudios han sido por lo general alentadores. Chrobak (2004, p. 16), reconoce esta tendencia proponiendo que:

[...] un estudiante es cognitivamente maduro y por ende exitoso, cuando sabe qué es comprender y cómo debe trabajar mentalmente para comprender, lo cual se logra a través del desarrollo de la metacognición. Además, el desarrollo de la metacognición nos hace tomar conciencia, por ejemplo, de que un párrafo es difícil de comprender y por eso controlamos la velocidad de lectura para, de esta manera, poder deducir el verdadero significado del escrito, con lo que el conocimiento de nuestra propia comprensión nos lleva a regular la actividad mental implicada en la comprensión, y es este aspecto el que ha tomado mayor importancia en las investigaciones más recientes.

A pesar de estos resultados positivos con respecto a la importancia de la metacognición en relación con el desempeño (Reynolds \& Miller, 2003; Pozo, 2003; Seeratan, 2002; Corno, 2001), otras investigaciones evidencian que algunos estudiantes, aun cuando contaban con estrategias metacognitivas, no las ponían en práctica en sus labores académicas, mostrando resultados contradictorios (Pintrich \& Zeidner, 2002; Dennis, 2002; Li y O'Neil, 1995). A partir de estos resultados, algunos hipotetizan que la puesta en práctica de las estrategias metacognitivas está mediada por la motivación del estudiante (Rotman \& Cross, 1990; Bergamo y Cornoldi, 1990). Este planteamiento se sustentó en la observación de que algunos alumnos, a pesar de contar con las herramientas metacognitivas para el éxito, fracasaban debido a una falta de motivación, presentando atribuciones externas de éxito, pesimismo y pocas expectativas asociadas al 
uso de las herramientas metacognitivas, teniendo por ende una menor puesta en práctica de estas. Así, muchos teóricos consideran hoy en día la motivación como un componente básico de la metacognición, sin el cual esta no se pone en marcha (Monereo, 2005; Rosendaal, Minneaert \& Boekaert, 2005).

El concepto de motivación se refiere a la tendencia general para alcanzar las metas u objetivos (Reategui \& Sattler, 1999). A pesar de sus diferentes concepciones, existe mucha evidencia de su influencia en el rendimiento académico (Sideridis, Botsas \& Morgan, 2006; Zimmerman, Kitsantas \& Campillo, 2005; Torrano \& González, 2004; Palinscar \& Brown, 2002). En la presente investigación se estudió la motivación académica a través de tres constructos con amplio sustento empírico, elaborando una medida basada en estas perspectivas. La primera perspectiva estudiada fue la motivación de logro, la cual se encuentra referida a la necesidad social, que conduce los esfuerzos y acciones de la persona, de manera persistente, a alcanzar estándares de excelencia (McClelland, Atkinson \& Clark, 1953). Las investigaciones con respecto a la motivación de logro encuentran que esta se relaciona positivamente con el desempeño académico (Veenman, 2002; Martínez \& Galán, 2000; Barbera \& Molero, 1996). La segunda perspectiva motivacional estudiada fueron las atribuciones causales referidas a la interpretación, interna o externa, que el individuo realiza respecto de los elementos responsables de un resultado, determinando su motivación futura (Weiner, 1992). También existe gran evidencia de su relación con el rendimiento académico (Dweck, 2002; Ugartexa, 2001; Buron, 1999). La tercera perspectiva estudiada es la autoeficacia, que se refiere a la creencia respecto a la propia capacidad que se posee para realizar una actividad con un resultado aceptable, determinando la extensión de sus esfuerzos (Bandura, 1999). Esta ha encontrado también gran relación con el desempeño académico (Castelló y Monereo, 2005; Zimmerman, 2002; Zimmerman \& Schunk, 2001; Hackett, 1995).

Desde sus inicios y por mucho tiempo, las investigaciones sobre metacognición se centraron en la población escolar. En esta última década, la educación superior ha empezado a ser el foco de muchos estudios. Este interés surge a partir de datos que demuestran una alta tasa de fracaso en los primeros años de educación universitaria alrededor del mundo, evidenciando que en muchos casos los alumnos en este nivel no han logrado aún un desarrollo metacognitivo óptimo.

En nuestro país es reciente el interés por evaluar las estrategias metacognitivas de nuestros alumnos, más aún de nuestros alumnos universitarios, quienes, se supone, deberían llegar a la universidad equipados y diestros en el uso de estas estrategias. 
La presente investigación se plantea la siguiente pregunta general de investigación: ¿Cuál es la relación entre las estrategias metacognitivas, la motivación académica y el rendimiento académico, en estudiantes ingresantes al primer nivel de estudios de una universidad de Lima metropolitana?

A partir de esta se desglosan las siguientes preguntas específicas: ¿cuál es la relación entre las estrategias metacognitivas y el rendimiento académico en estudiantes ingresantes al primer nivel de estudios de una universidad de Lima metropolitana?, ¿cuál es la relación entre la motivación académica y el rendimiento académico en estudiantes ingresantes al primer nivel de estudios de una universidad de Lima metropolitana?, ¿cuál es la relación entre las estrategias metacognitivas y la motivación académica en estudiantes ingresantes al primer nivel de estudios de una universidad de Lima metropolitana?

\section{MÉtodo}

El método utilizado fue el descriptivocorrelacional, con el propósito de evidenciar y describir las relaciones entre los puntajes de las variables (estrategias metacognitivas, motivación académica y rendimiento académico), en alumnos ingresantes a una universidad en Lima metropolitana (Hernández, FernándezCollado \& Baptista, 2007). El diseño utilizado fue descriptivo-correlacional, buscando determinar la correlación entre las variables estudiadas.

\section{PARTICIPANTES}

La población objetivo estuvo constituida por todos los alumnos matriculados en el primer ciclo de estudios generales de las facultades de Medicina y Veterinaria de una universidad privada de Lima metropolitana, en el año académico 2007-I. Esta fue de 190 estudiantes, 103 mujeres y 87 varones. La muestra de 156 estudiantes se obtuvo a partir de un muestreo no probabilístico circunstancial, con 85 mujeres y 71 varones.

\section{INSTRUMENTOS}

\section{Evaluación de la motivación académica}

Se elaboró la Escala de Motivación Académica, la cual presenta 20 ítems constituidos como frases, para evaluar a través de una escala Likert. Su aplicación se realiza con estudiantes universitarios, tanto de manera individual como colectiva, y tomó 15 minutos aproximadamente. Sus resultados reflejan puntajes en las siguientes tres escalas:

- Autoeficacia académica: evalúa la percepción que el alumno tiene de su propia capacidad para desempeñarse con éxito en las tareas académicas. 
- Atribuciones internas de logro: evalúa las explicaciones que el alumno genera con respecto a la causa de sus resultados académicos.

- Acciones orientadas al logro: evalúa las conductas del estudiante que lo orientan a la consecución del éxito en tareas evaluadas con estándares de excelencia.

El proceso de creación de la Escala de Motivación Académica se inició con la revisión de las conceptualizaciones vigentes para cada una de las perspectivas motivacionales estudiadas (Weiner, 1992; Bandura, 1993; McClelland, 1958) y creación de ítems para cada una de ellas. Luego se trabajó la validación de la redacción de los ítems con la ayuda voluntaria de siete ingresantes a una universidad en Lima metropolitana. Seguidamente, se procedió a la validación por seis jueces, quienes analizaron su contenido y redacción, aplicándose el coeficiente $\mathrm{V}$ de Aiken para conocer el grado de adecuación del ítem al factor, siendo un ítem eliminado por presentar una relación menor a 0.5. Finalmente, se realizó una aplicación piloto, con 74 alumnos ingresantes a una universidad privada en Lima metropolitana, lográndose un coeficiente Alpha de Cronbach de 0.79.

\section{Evaluación de las estrategias metacognitivas}

Para la evaluación de las estrategias metacognitivas se aplicó el Inventario de Estrategias Metacognitivas - Estado (State Metacognitive Inventory) elaborado por Harold O'Neil y Jamal Abedi en 1996 en la Universidad del sur de California y posteriormente traducido al español y aplicado en estudiantes universitarios por Martínez Fernández en la Universidad de Barcelona (2001). Se trata de un inventario de frases evaluadas en escala Likert, cuya aplicación puede ser individual o colectiva, tomando esta aproximadamente 15 minutos . El inventario encuentra puntajes en las siguientes cuatro escalas: conocimiento de estrategias cognitivas, donde evalúa el conocimiento de las estrategias cognitivas que el alumno utiliza ante una tarea para el logro de su meta; planificación metacognitiva, donde evalúa el uso de estrategias para el reconocimiento de las demandas de una tarea, la identificación del objetivo y la elaboración de un plan para lograrlo; monitoreo metacognitivo, donde evalúa el uso de estrategias de chequeo continuo y regulación, durante la puesta en práctica del plan para el logro del objetivo; $\mathrm{y}$, por último, consciencia metacognitiva, donde se valúa el nivel de conciencia que el individuo presenta con respecto a su ejecución estratégica.

Los estudios de validación del inventario demuestran su validez de constructo a través del análisis de componentes principales con rotación Varimax, revelaron cuatro factores que corresponden a la estructura teórica del instrumento. Asimismo, para la muestra 
estudiada en la presente investigación se obtuvo un elevado coeficiente de confiabilidad Alpha de Cronbach (0.88).

\section{Rendimiento académico}

Como medida del rendimiento académico se utilizaron los promedios ponderados finales del ciclo académico 2007-I, de los estudiantes que conformaron la muestra. Estos puntajes han sido elegidos como instrumentos para la medición del rendimiento académico, ya que se trata de una medida que resume el desempeño del estudiante a lo largo del semestre universitario. Además, históricamente, los investigadores interesados en el estudio de la motivación y su influencia en el rendimiento académico y aprendizaje, han utilizado el rendimiento académico como medida de contraste para observar los efectos de su instrucción. Igualmente, los estudios para considerar los efectos de la instrucción metacognitiva, utilizan esta variable como medida de su impacto.

En ambas facultades, Medicina y Veterinaria, los estudiantes cursan durante el primer semestre cinco asignaturas obligatorias para su carrera, que hacen un total de 18 créditos académicos. De los cinco cursos que llevan los estudiantes en este primer ciclo, cuatro son cursos comunes para medicina y veterinaria (Comunicación y Aprendizaje, Química, Matemática y Ecología) teniendo el mismo sílabus e incluso los mismos profesores. Así, el único curso distinto para estos alumnos es el de Primeros Auxilios para los de la Facultad de Medicina y Bases Biológicas de las Ciencias Veterinarias, para los de la Facultad de Veterinaria, ambas asignaturas de dos créditos cada una.

\section{RESULTADOS Y DISCUSIÓN}

Para la realización del contraste de hipótesis específicas se trabajó con el estadístico de correlación Pearson, puesto que las medidas de estrategias metacognitivas, motivación académica y rendimiento académico, cumplen con los supuestos necesarios para su aplicación.

El análisis de la dirección y fuerza de las correlaciones obtenidas se interpretó de acuerdo con lo propuesto en la tabla 1 (Castro \& Galindo, 2000).

Tabla 1

Interpretación de la fuerza y dirección de la correlación

\begin{tabular}{cc}
\hline $\begin{array}{c}\text { Coeficiente } \\
\text { de correlación }\end{array}$ & $\begin{array}{c}\text { Grado de } \\
\text { correlación }\end{array}$ \\
\hline Mayor a 0.65 & Alto \\
$0.35-0.65$ & Mediano \\
Menor a 0.35 & Bajo \\
\hline
\end{tabular}


Tabla 2

Correlación Pearson entre estrategias metacognitivas

y el rendimiento académico

\begin{tabular}{ll}
\hline & $\begin{array}{c}\text { Motivación académica } \\
\mathbf{r}\end{array}$ \\
\hline Estrategias metacognitivas & $0.15^{*}$ \\
Conciencia & 0.10 \\
Planificación & $0.14^{*}$ \\
Monitoreo & $0.16^{*}$ \\
Estrategias cognitivas & 0.11 \\
\hline
\end{tabular}

$N=156 \quad * p<.05$

El análisis de correlación de Pearson revela la existencia de correlaciones positivas y significativas, entre el rendimiento académico y los puntajes de la prueba en su totalidad. En cuanto a las escalas que componen la prueba se halla correlación significativa entre la escala de monitoreo y la escala de planificación, con el rendimiento académico. Así, las demás escalas no logran correlaciones suficientemente fuertes para ser significativas. Los resultados coinciden con la literatura que encuentra relación entre la metacognición y el desenvolvimiento académico (Zimmerman, 2002; Corno, 2001; Weinstein, Husman \& Dierking, 2000; Castelló \& Monereo, 2000; García \& La Casa, 1990, etcétera). Sin embargo, difieren en el nivel de correlación encontrado, sumándose a las investigaciones que evidencian un nivel bajo de relación entre las dos variables (Boekaerts, Els de Koning \& Vedder, 2006;
Norton, 2004; Pintrich, 2000; Yap \& Van Der Leij, 1993). Algunos autores han propuesto que esto se debe a que existe gran variabilidad en la puesta en práctica de estrategias metacognitivas, debido a variaciones en la motivación (Rottman \& Cross, 1990).

Por otro lado, el hecho de que las escalas de planificación y de monitoreo logren correlaciones algo mayores $\mathrm{y}$ significativas, con el rendimiento académico respalda lo planteado por investigadores que demuestran que el componente de regulación metacognitiva es el más importante para el desempeño y aprendizaje significativo (Torrano \& González, 2004; Zimmerman \& Schunk, 2001; Pintrich, 2000, Corno, 2001).

Por último, no se evidencian diferencias en la aplicación de las estrategias metacognitivas según el género ni la facultad. En cuanto al género, este resultado concuerda con muchas otras investigaciones (Martínez-Fernández, 
2004; Pajares \& Schunk, 2002; Borkowski, 1992). En cuanto a la facultad, se puede plantear la posibilidad de que los procesos empleados por la universidad en cuestión han permitido seleccionar estudiantes con un nivel metacognitivo similar. Se puede también pensar que, al no encontrarse diferencias según el género ni según la facultad, se trata del nivel metacognitivo en el que se encuentran los jóvenes en nuestro medio, al egresar de la secundaria.

El análisis estadístico demostró que existen correlaciones positivas y significativas entre los puntajes totales de la escala de motivación académica y el rendimiento académico de los alumnos. Asimismo, se observan correlaciones positivas y significativas entre los puntajes de todos los factores de la escala de motivación académica con el desempeño académico. De esta manera, se observa que los puntajes elevados en las escalas de autoeficacia, atribución y acciones de logro correlacio- nan de manera significativa y positiva con un rendimiento académico alto. Estos resultados se unen a una larga trayectoria de estudios que confieren a la motivación un papel importante en el aprendizaje y los resultados académicos (Turner, Meyer \& Schweinle, 2003; Rosendaal, Minneaert \& Boekaerts, 2005; Butler \& Winne, 1995). Las atribuciones resaltan como uno de los factores más relacionados al rendimiento, coincidiendo con investigaciones previas, que demuestran su importancia (Crowson, Duke \& Akey, 2004; Borkowski, Weyhing \& Carr, 1991).

También se puede observar que la motivación logra una asociación más elevada y significativa con el desempeño, que las estrategias metacognitivas $(r=0.353$ vs. $r=0.159)$. Esto coincide con lo encontrado por Wentcel (2003) y Borkowski (1992), quienes concluyen que la motivación interviene de manera significativa en el rendimiento académico, proponiendo

Tabla 3

Correlación Pearson entre la motivación académica y el rendimiento académico

\begin{tabular}{ll}
\hline & $\begin{array}{c}\text { Rendimiento académico } \\
\mathbf{r}\end{array}$ \\
\hline Motivación académica & $.35^{* * *}$ \\
Autoeficacia académica & $.18^{*}$ \\
Atribuciones internas de logro & $.39^{* * *}$ \\
Acciones orientadas al logro & $.26^{* *}$ \\
\hline
\end{tabular}

$N=156$

${ }^{*} p<.05 \quad{ }^{* *} p<.01 \quad{ }^{* * *} p<.001$


un efecto mediador de la práctica de las estrategias metacognitivas. Por otro lado, los estudiantes no se diferencian en su nivel de motivación académica según su género, pero sí según su facultad, encontrándose los de medicina más motivados que los de veterinaria. En cuanto al género, coincide con investigaciones anteriores, donde los niveles de motivación son similares entre varones y mujeres (Thornberry, 2003; Morante, 1984). La diferencia motivacional entre facultades coincide con la diferencia observada en el rendimiento académico, pues en este no se encuentran diferencias según el género, pero sí según la facultad, siendo los estudiantes de medicina aquellos con mejores promedios. A partir de esa evidencia se puede observar que la motivación juega un rol importante en el rendimiento académico de los estudiantes.

Tabla 4

Correlación Pearson entre los puntajes totales de las pruebas de estrategias metacognitivas y motivación académica

Motivación académica

$r$

Estrategias $38^{\star * *}$

metacognitivas

$N=156 \quad * * * p<.001$

La tabla 4 evidencia una correlación significativa entre el uso de estrategias metacognitivas y la motivación académica en los estudiantes universitarios que cursan el primer ciclo de estudios.

La tabla 5 evidencia la existencia de correlaciones positivas y significativas entre todos los factores de ambas pruebas, evidenciando su asociación. Estos resultados confirman la asociación que diversos autores realizan entre la aplicación de las estrategias metacognitivas y la motivación académica del estudiante (Torrano \& González, 2004; Martínez-Fernández, 2004; Crowson, Duke \& Akey, 2004; Veenmann, 2002; Wigfield \& Eccles, 2002; Ugartexa, 2001; Baker \& Cerro, 2000).

Como se observa en la tabla 6 , la relación entre las tres medidas estudiadas es baja, evidenciando solo un $12,7 \%$ de nivel de predicción como efecto conjunto de la motivación académica y estrategias metacognitivas. Al observar los puntajes Beta obtenidos en este análisis se encuentra que las estrategias metacognitivas logran niveles que no se diferencian del cero, mientras que la motivación académica logra puntajes Beta mayores. Esta evidencia unida a lo encontrado en los análisis de las hipótesis anteriores, en los que se observó que los resultados de las estrategias metacognitivas presentaban correlaciones muy bajas con el rendimiento, mientras que la motivación académica presentaba correlaciones medias, se procedió a realizar nuevamente el análisis de regresión lineal, esta vez solo considerando el 
Tabla 5

Correlación Pearson entre cada una de las escalas de las pruebas de estrategias metacognitivas y de motivación académica

\begin{tabular}{lccc}
\hline & Autoeficacia & \multicolumn{2}{c}{ Atribución Acciones de logro } \\
& $\mathbf{r}$ & $\mathbf{r}$ & $\mathbf{r}$ \\
\hline Conciencia & $.27^{* *}$ & $.24^{* *}$ & $.40^{* *}$ \\
Planificación & $.18^{*}$ & $.22^{* *}$ & $.34^{* * *}$ \\
Monitoreo & $.28^{* * *}$ & $.25^{* *}$ & $.30^{* * *}$ \\
Estrategias cognitivas & $.17^{*}$ & $.17^{*}$ & $.32^{* * *}$ \\
\hline
\end{tabular}

$N=156 \quad{ }^{*} p<.05 \quad{ }^{* *} p<.01 \quad{ }^{* * *} p<.001$

Tabla 6

Regresión lineal y múltiple para los puntajes totales de estrategias metacognitivas, motivación académica y rendimiento académico

\begin{tabular}{|c|c|c|c|c|}
\hline $\mathbf{R}^{2}$ & $\mathbf{F}$ & & B & \\
\hline \multirow{2}{*}{.127} & \multirow{2}{*}{$11.023^{\star *}$} & Constante & $\begin{array}{c}\text { Estrategias } \\
\text { metacognitivas } \\
\text { total }\end{array}$ & $\begin{array}{l}\text { Motivación } \\
\text { académica } \\
\text { total }\end{array}$ \\
\hline & & 10.761 & 0.005 & $0.12^{* \star}$ \\
\hline
\end{tabular}

Tabla 7

Regresión lineal simple para los puntajes totales de estrategias metacognitivas, motivación académica y rendimiento académico

\begin{tabular}{cccc}
\hline $\mathbf{R}^{2}$ & $\mathbf{F}$ & $\mathbf{B}$ \\
\hline 0.126 & 22.080 & Constante & $\begin{array}{c}\text { Motivación } \\
\text { académica total }\end{array}$ \\
\cline { 3 - 4 } & & 10.926 & $0.12^{* *}$ \\
\hline $\mathrm{N}=156 \quad{ }^{* *} \mathrm{p}<.01$ & & &
\end{tabular}


puntaje total de la Escala de Motivación Académica (tabla 7).

Como resultado de este análisis, se observó que los puntajes totales del inventario de Motivación Académica logran predecir por sí mismos -en un $12,6 \%$ - el rendimiento académico. De esta manera se comprueba lo que se venía observando: que los puntajes de motivación académica son los que en realidad se encuentran generando la predicción del desempeño.

Por último, con la finalidad de conocer el potencial máximo de predicción de las escalas de motivación académica se llevó a cabo el análisis de regresión lineal, considerando solo dichas escalas.

A partir de este análisis (tabla 8) se observa que la regresión lineal llevada a cabo a partir de la consideración de cada una de las escalas de motivación académica logra un 19,3\% de predic- ción del rendimiento académico de los estudiantes en la muestra. Así, se evidencia que las escalas de la prueba logran mayores niveles predictivos del desempeño que los resultados totales, mientras que la escala de atribuciones internas de logro es la que mayor peso aporta a la predicción del rendimiento académico.

Con estos resultados se concluye que la motivación académica sí resulta predictiva del rendimiento académico, mientras las estrategias metacognitivas no lo hacen. Este resultado apoya lo encontrado por diversos autores con respecto a la fuerza de la relación entre la motivación académica y el rendimiento, así como la baja relación entre la metacognición y el desempeño (Sideridis, Morgan \& Botsas, 2006; Wigfield \& Eccles, 2002; Pintrich \& Schunk, 2002; Eyring, 1995).

Tabla 8

Regresión lineal múltiple para los puntajes por escalas de motivación académica y rendimiento académico

\begin{tabular}{|c|c|c|c|c|c|}
\hline $\mathbf{R}^{2}$ & $F$ & \multicolumn{4}{|c|}{ B } \\
\hline \multirow{2}{*}{.193} & \multirow{2}{*}{$12.066^{* * *}$} & Constante & $\begin{array}{l}\text { Autoeficacia } \\
\text { académica }\end{array}$ & $\begin{array}{c}\text { Estrategias } \\
\text { metacognitivas } \\
\text { total }\end{array}$ & $\begin{array}{c}\text { Motivación } \\
\text { académica } \\
\text { total }\end{array}$ \\
\hline & & 11.487 & $-0.17^{*}$ & $0.38^{* * *}$ & $0.12^{*}$ \\
\hline & $p<.05$ & $p<.01$ & ${ }^{* *} p<.001$ & & \\
\hline
\end{tabular}




\section{REFERENCIAS}

Baker, L. \& Cerro, L. (2002). Assessing metacognition in children and adults. En Schraw, G. \& Impara, J. (Eds.). Issues in the measurement of metacognition (pp. 99-145). Lincoln, NE: Buros Institute of Mental Measurements, University of Nebraska.

Bandura, A. (1999). Self-efficacy: The exercise of control. Nueva York: Freeman.

Bandura, A. (1993). Perceived selfefficacy in cognitive development and functioning. Educational Psychologist, 28.

Barberá, E. \& Molero, C. (1996). Motivación social. En Garrido, I. (Ed.). Psicología de la motivación. Madrid: Síntesis.

Bergamo, E. \& Cornoldi, C. (1990). Metacognitive control processes and memory deficits in poor comprehenders. Learning Disability Quarterly, 13.

Boekerts, M.; Els de Koning \& Vedder, P. (2006). Goal directed behavior and contextual factors in the classroom: an innovative approach to the study of multiple goals. Educational Psychologist 41, (1)

Borkowski, J. G. (1992). Metacognitive theory: a framework for teaching literacy, writing and math skills. Journal of Learning Disabilities, 25.

Borskowski, J. G.; Weyhing, R. S. \& Carr, M. (1991). Classroom Applications of Mnemonic Instruction: Acquisition, maintenance, and generalization. Exceptional Children, 9.

Buron, J. (1999). Motivación y aprendizaje. Bilbao: Martínez Roca.

Butler, D. L. \& Winne, PH. H. (1995). Feedback and self-regulated learning: a theoretical synthesis. Review of Educational Research, 65, (3)

Castello, M., \& Monereo, C. (2000). Las concepciones de los profesores sobre la enseñanza de estrategias de aprendizaje. Ensayos y Experiencias, 6 (33).

Castello, M., \& Monereo, C. (2005). Student's note taking as a knowledge construction tool. Educational Studies in Language and Literature, 5, (3)

Castro, J. \& Galindo, M. (2000). Estadística multivariable: Análisis de correlaciones. Salamanca: Amaru Ediciones.

Chrobak, R. (2004). La metacognición y el mejoramiento de la enseñanza de química universitaria. Las Ciencias, 22 (1).

Corno, L. (2001). Volitional aspects of self-regulated learning. En Zimmerman, B. J. \& Schunk, D. H. (Eds.). Self-regulated learning and 
academic achievement. Nueva Jersey: Erlbaum.

Covington, M. (2000). Goal theory, motivation, and school achievement: an integrative review. Annual Review of Psychology, 51.

Crowson, M.; Duke, L. \& Akey, L. (2004). Predicting High School Students' Cognitive Engagement and Achievement: Contributions of Classroom motivation. Contemporary Educational Psychology, 29, (4).

Dennis, D. (2002). Metacognition and Reflection. Educational Psychology, 30 .

Dweck, C.S. (2002). Messages that motivate: How praise molds student's beliefs, motivation and performance. En Aronson (Ed.). Improving academic achievement: Impact of psychological factors on education. San Diego: Academic Press.

Eyring, H. (1995). On becoming a disciple-scholar: Lectures presented at the Brigham Young University Honors Program (Discipline and discipleship lecture series).

Flores, R. (2000). Autorregulación, metacognición y evaluación. Acción Pedagógica, 9(1).

García, J. \& La Casa, P. (1990). Procesos Cognitivos Básicos. Años Escolares. En Palacios, J.; Marchesi, A. \& Coll, C. (Comp.). Desarrollo Psi- cológico y Educación. Tomo I: Psicología Evolutiva, Cap. XV. Madrid: Alianza Editorial, S. A.

García, E. (2001). Estilos de aprendizaje y cognitivos. En Puente, A. (Ed.). Estilos de aprendizaje y enseñanza. Madrid: CEPE.

Hackett, G. (1995). The role of mathematics self-efficacy in the choice of math-related majors of college women and men: A path analysis. Journal of Counseling Psychology, 32.

Hernández, R.; Fernández-Collado, C. \& Baptista, P. (1998). Metodología de la investigación. Santa $\mathrm{Fe}$ de Bogotá: McGraw-Hill.

Kuhl, J. (2001). A functional approach to motivation: The role of goalenactment and self-regulation in current research on approach and avoidance. En Efklides, A.; Kuhl, J. \& Sorrentino; R. M. (Eds.). Trends and prospects in motivation research (pp. 239-268). Dordrecht, The Netherlands: Kluwer.

Li, L. C. \& O'Neil, H. F. (1995). The effects of effort and worry on distance learning. Manuscript submitted for publication. National Open University of Taiwan (LCL) and the university of Southern California (HON).

Martínez-Fernández, R. (2001). Concepción de aprendizaje y estrategias metacognitivas en estudiantes universitarios de psicología. Anales de 
psicología 2007,23, (1).

Martínez-Fernández, J. R. (2004). Concepción de aprendizaje, metacognición y cambio conceptual en estudiantes universitarios de psicología. Tesis para optar el grado de doctor. Departamento de Psicología Básica. Barcelona: Universidad de Barcelona.

Martínez, J. R. \& Galán, F. (2000). Estrategias de aprendizaje, motivación y rendimiento académico en alumnos universitarios. Revista Española de Orientación y Psicopedagogía, 11, (1).

Mayer, R. E. (2003). Learning and instruction. Upper Saddle River. Nueva Jersey: Pearson.

McClelland, D.; Atkinson, J.; Clark, R. $\&$ Lowell, E. (1958). The achievement motive. Nueva York: Appleton-Century-Clofts.

Meltzet, L. J. (1993). Strategy use in students with learning disabilities: The challenge of assessment. En Meltzer, L. J. (Ed.). Strategy assessment and instruction for students with learning disabilities. Austin: Pro-Ed.

Monereo, C. (2005). Internet y competencias básicas. Barcelona: Graó.

Morante, C. (1984). Motivación de logro y rendimiento escolar en alumnos de doce a dieciséis años pertenecientes a dos colegios reli- giosos de Lima. Tesis para optar el grado de bachiller en psicología. Lima: Pontificia Universidad Católica del Perú.

Muria, S. (2004). Indicadores de diagnóstico para la implementación de una web geométrica con alumnos deficientes auditivos en aulas inclusivas. Tesis para optar el grado de doctor. Barcelona: Universitat de Barcelona.

Norton, L. (2004). Using assesment criteria as learning criteria: a case study in psychology. Assesment and Evaluation in Higher Education, 29, (6).

O’Neil, H. F. \& Abedil, J. (1996). Reliability and validity of a state metacognitive inventory: Potential for alternative assessment. Journal of Educational Research, 89, (4).

Pajares, F. \& Schunk, D. H. (2002). Self and self-belief in psychology and education: A historical perspective. En Aronson (Ed.). Improving academic achievement: Impact of psychological factors on education. San Diego: Academic Press.

Palincsar, A. S. \& Brown, A. L. (2002). La enseñanza para la lectura autorregulada. En Resnick, L y Klopfer, L. (Comp.). Currículum y cognición. Buenos Aires: Aique.

Pintrich, P. R. (2000). The role of goal orientation in self-regulated learning. En Boekaerts, M.; Pintrich, P. R. \& Zeidner, M. (Eds.). Handbook 
of self-regulation. San Diego: Academic Press.

Pintrich, P. R., \& Schunk, D. H. (2002). Motivation in education: Theory, research, and applications (2. ${ }^{\mathrm{a}} \mathrm{ed}$.). Upper Saddle River, NJ: Merrill Prentice Hall.

Pintrich, P. R. \& Zeidner, M. (2002). Handbook of self-regulation. Elsevier: Academic Press.

Pozo, J. I. (2003). Adquisición de conocimiento. Madrid: Morata.

Reátegui, N. \& Sattler, C. (1999). Metacognición. Estrategias para la construcción del conocimiento, $\left(2 .^{\mathrm{a}}\right.$ ed.). Lima: Cedum.

Reynolds, W. M. \& Miller, G. E. (2003). Current perspectivs in educational psychology. En Reynolds, W. M. \& Miller, G. E. (Eds.). Handbook of psychology: Educational psychology. Nueva Jersey: Wiley.

Ronmainville, R. (1994). Awareness of cognitive strategies: The relationship between university students' metacognition. Studies in Higher Education, 19, (3).

Rosendaal, J. S.; Minneart, A. \& Boekaerts, M. (2005). The influence of teacher perceived administration of self-regulated learning on student's motivation and information-processing. Learning and Instruction, 15, (2).
Rottman, T. R., \& Cross, D. R. (1990). Using informed strategies for learning to enhance the reading and thinking skills of children with learning disabilities. Journal of Learning Disabilities, 23, (5).

Sandia, L. (2004). Metacognición en niños: una posibilidad a partir de la teoría Vygotskiana. Revista de Acción Pedagógica, 2.

Seeratan, K. L. (2002). Learning disabilities: The inter-relational dynamics of the cognitive, metacognitive and self systems. Documento de trabajo presentado en la Jean Piaget International Society Conference, Philadelphia, Pennsylvania.

Siderdis, G.; Morgan, P. \& Botsas, G. (2006). Predicting LD on the basis of metacognition motivation and psychopathology: an ROC analysis. Journal of Learning Disabilities, 39 , (3).

Thornberry, G. (2003). Motivación de logro en estudiantes de secundaria de colegios de diferente gestión. Tesis para optar el título de licenciada en psicología. Lima: Universidad de Lima.

Torgesen, J. (1994). Issues in the assessment of executive function: An information processing perspective. En Lyon, G. R. (Ed.). Frames of reference in the assessment of learning disabilities: New views on measurement issues. Toronto: 
Brooks.

Torrano, F. \& González-Torres, M. C. (2004). El aprendizaje autorregulado: presente y futuro de la investigación. Revista Electrónica de Investigación Psicoeducativa, 2(1).

Turner, J.; Meyer, D. \& Schweinle, A. (2003). The importantce of emotion in theories of motivation: empirical, methodological and theoretical considerations from a goal theory perspectiva. International Journal of Educational Research, 39.

Ugartetxea, J. (2001). Motivación y metacognición, más que una relación. Revista Electronica de Investigación y Evaluación Educativa, 7, (2). Consultado el 24 de Septiembre del 2007. En <http://www.uv.es/ RELIEVE/v 7n 2/RELIEVEv 7n21.htm>.

Veenmam, M. (2002). Changes in the relation between cognitive and metacognitive skills during the acquisition of expertise. European Journal of Psychology of Education, 14, (4).

Weiner, B. (1992). Human motivation: Metaphors, theories, and research. Newbury Park, CA: Sage.

Weinstein, C. E.; Husman, J. \& Dierkiing, D. R. (2000). Self-regulation interventions with a focus on learning strategies. En Boekaerts, P. R.; Pintrich, P. R. \& Zeidner, M. (Eds.). Handbook of self-regulation.
San Diego: Academic Press.

Wentcel, K. (2003). School adjustment. En Reynolds, W. y Miller, G. (Ed.). Handbook of Psychology: Educational Psychology. Nueva Jersey: Wiley.

Wigfield, A. \& Eccles, J. S. (2002). Facilitating children's motivation to read. En Baker, L.; Dreher, M. J. \& Guthrie, J. T. (Eds.). Engaging young readers (pp. 140-158). Nueva York: Guilford.

Winne, H. P. (2005). Key issues in modeling and applying research on self-regulated learning. Applied Psychology, 54(2).

Wong, B. Y. L. (1992). On cognitive process-based instruction: An introduction. Journal of Learning Disabilities, 25, (3).

Yap, R., \& Van Der Leij, A. (1993). Word processing in dyslexics: An automatic decoding deficit? Reading and Writing: An Interdisciplinary Journal, 5.

Zimmerman, B. J. \& Schunck, D. H. (2001). Self-regulated learning and academic achievement: Theoretical perspectives. Nueva Jersey: Erlbaum.

Zimmerman, B. J. (2002). Becoming self-regulated learner: An overview. Theorye into Practice, 41.

Zimmerman, B. J.; Kitsantas, A. \& Campillo, M. (2005). Evaluación de la au- 